\title{
Implementation of PDMS-based Slanted Edge-type Microelectrode Array for Neural Stimulation ${ }^{1}$
}

\author{
Jun-Min $\mathrm{Kim}^{1}$ and Sun-Hyung Kim${ }^{2 *}$ \\ ${ }^{1}$ Medical Device Research Center Medical \& Health Device Division, $5^{\text {th }}$ Floor, \\ Korea Testing Laboratory, 87, Digital-ro 26-gil, Guro-gu, Seoul, \\ Republic of Korea \\ ${ }^{2}$ Dept. of Information \& Communication Eng., Graduate Soonchunhyang Univ., \\ Chungnam, Republic of Korea \\ 1junmin83@gmail.com, ${ }^{2}$ shkim@sch.ac.kr
}

\begin{abstract}
PDMS-based multi-electrodes are widely used in acute experiments for neural interfaces, and generally have a vertical-edge electrode structure. Previous studies have shown that when stimulating neural tissue with a vertical-edge electrode, a high current density is produced at the edge of the electrode, which makes uniform stimulation difficult. However, slanted-edge electrodes can induce a relatively uniform current density distribution. PDMS is a very stable material, physically and chemically; thus, it is difficult to produce slanted-edge electrodes because no efficient etching process exists. In order to address this problem, we propose a process to fabricate sloped structures using the under-exposure characteristics of photolithography, and to fabricate sloped holes efficiently in PDMS by using it as a cast structure. The validity of the proposed process was verified by fabricating vertical-edge, slanted-edge, and surface-mounted edge electrodes. We verified the current density distribution according to the electrode structure by simulation, and applied signals to vertical-edge and slanted-edge samples to observe the current leakage. It has been confirmed that the slanted-edge structure improves the stability and reliability of the electrode.
\end{abstract}

Keywords: Slanted-edge electrode, PDMS, Neural stimulation, photolithography, current density distribution, current leakage

\section{Introduction}

Brain-computer interfaces (BCI) are a technology that invasively or non-invasively accesses the functions of the nervous system using various engineering techniques to record biological signals or stimulate the neurons [1]. To record a clear signal (which has high signal to noise ratio) or stimulate neurons in high resolution, electrodes are inserted directly into the tissue. This has the advantage of high spatial resolution, but can produce fatal consequences such as bacterial infection [2]. Non-invasive methods are safe but have low spatial resolution [3]. Recently, minimally invasive surgery has been widely performed to minimize the surgical area and the distance between the electrodes and the neural tissue [4].

The electrodes used for BCI have gradually changed from needle-type to planar-type in order to minimize damage to nerve tissue, and to facilitate placement as close as possible to the target nerve $[3,5]$. The electrodes have a form in which the entire device is inserted

1 Abbreviations: Polydimethylsiloxane (PDMS); Brain-computer interface (BCI); tetrabutylammonium fluoride (TBAF); hydroxyethyl methacrylate (HEMA); buffered oxide etchant (BOE)

Received (October 29, 2017), Review Result (December 8, 2017), Accepted (December 11, 2017) 
into the body to prevent bacterial infection. As a result, the biocompatibility and physical properties of the electrode have become important factors [6, 7].

Polymer-based electrodes have been recently studied for these reasons [8-12]. These electrodes are commonly fabricated with an insulator-conductor-insulator sandwich-like structure. Polyimide and parylene polymers are widely used as the insulators owing to their high biocompatibility and easy fabrication process. However, PDMS has attracted much attention as the base material for inserted electrodes because it has excellent biocompatibility and its Young's modulus is very similar to that of nerve tissue [10, 12]. It is used for medical applications and food additives, and is certified by the FDA. However, because of its extreme physical and chemical stability, subjecting it to semiconductor processes such as metal deposition or etching is difficult [13].

Electrical stimulation of nerve tissue through electrodes is closely related to the current density. If excessive current is applied to the nerve tissue, neural tissue can be damaged. In addition, unwanted neural tissue can be stimulated, and side effects can occur. It has been theoretically proven that the current density distribution varies with the aperture shape of the electrode [14-16].

There are three major tissue damage problems caused by neural electrodes. The first is that the tissue can be stressed by the implanted electrode, or damaged during the surgical procedure. The second is that nerve tissue is damaged by irreversible electrochemical reactions at the interface of the electrode and tissue. Finally, long-term excitation of nerve tissue by excessively high frequency and amplitude stimulation causes permanent damage $[17,18]$.

When invasive electrodes were used, damage caused by surgical procedure was difficult to avoid; thus, optimizing surgical techniques is the best way to minimize tissue damage. However, the other issues can be minimized by optimizing the stimulation electrode. The most efficient method is to stabilize the electrical stimulation by making the current density distribution of the electrode uniform.

When the wall between the electrode and the insulator (PDMS) is vertical or the electrode is surface-mounted, the distribution of the current density is concentrated on the electrode edge. Conversely, when the wall is slanted, the current density is more evenly distributed. Therefore, it is possible to manufacture a safe and effective stimulation electrode by applying this principle to PDMS-based planar electrodes. However, research on the fabrication of holes having a slope in the PDMS film has been rarely reported [11]. Therefore, we propose an effective fabrication scheme for sloped structures, and the current density distribution of the fabricated electrodes was simulated using COMSOL. In addition, to estimate the durability of the electrodes in a human body, reliability experiments were performed in vitro.

\section{Materials and Methods}

\section{A. Fabrication Process of Proposed Slanted Electrode}

The overall process is summarized as follows. A thin PDMS film is formed on a silicon wafer, and a thin gold film is deposited. After forming a mask through a photolithography process, a gold wire is formed by wet etching. A photoresist sacrificial post is then fabricated on the electrode, and covered with another PDMS layer. Then, the surface of the PDMS is etched by about $1 \mu \mathrm{m}$ using a tetrabutylammonium fluoride (TBAF) solution and the sacrificial post is removed. Finally, the outline of the electrode is cut using a ND:YVO4 laser[12]. 


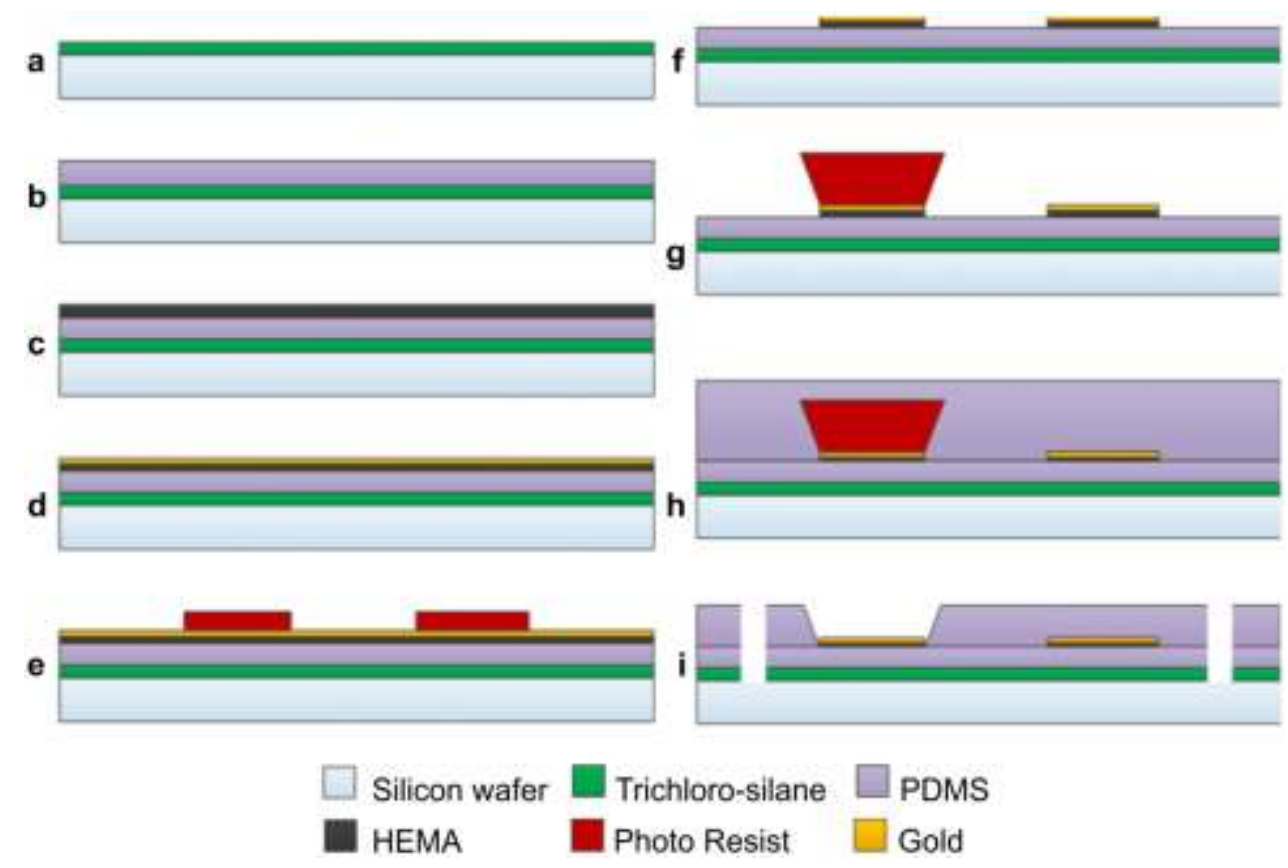

Figure 1. Fabrication process of slanted-edge electrode[12]: (a) form selfassembled monolayer trichlorosilane on the wafer; (b) spin-coat PDMS at $1000 \mathrm{rpm}$; (c) polymerize hydroxyethyl methacrylate (HEMA) on the PDMS layer to increase the adhesion; (d) deposit Ti (not shown in Fig) and Au; (e) pattern the metal layer using photolithography; (f) etch the metal layer; $(g)$ form the sacrificial post with a slanted wall using the underexposure characteristics of a negative photoresist; (h) spin-coat with PDMS and cure; and (i) wet etch the PDMS with tetrabutylammonium fluoride (TBAF) solution and cut the device outline using laser ablation

The detailed process sequence is shown in Figure 1. First, silicon wafers were prepared by a standard cleaning process. Then, trichlorosilane $(0.3 \mathrm{ml})$ was placed in an evaporation dish, and placed in a vacuum desiccator next to a silicon wafer, onto which a self-assembled monolayer was transferred by evaporation (Figure 1a). Vacuum was maintained for $4 \mathrm{~h}$ at a level of 600 Torr by using a vacuum pump (Rocker 300, Rocker Scientific Co., Taiwan). PDMS (Sylgard ${ }^{\circledR} 184$, Dow Corning, USA) was mixed with the curing agent in a weight ratio of 10:1, and any bubbles generated were removed by centrifuging (Centrifuge 5804R, Eppendorf International, Germany) for $3 \mathrm{~min}$ at 3000 rpm.

The resultant bubble-free PDMS solution was spin-coated on a silicon wafer that was coated with a trichlorosilane self-assembled monolayer at $1000 \mathrm{rpm}$ for $30 \mathrm{~s}$. Then it was cured in a $75{ }^{\circ} \mathrm{C}$ oven for $4 \mathrm{~h}$, to form a $\sim 70$ - $\mu$ m-thick PDMS film on the silicon wafer (Figure 1b). The surface of the PDMS was modified to be hydrophilic in order to stably deposit gold. Reactive ion etching (RIE) (Oxford Instruments, USA) with oxygen plasma at 75 mTorr and flow rate of $20 \mathrm{sccm}$ was then performed, before hydroxyethyl methacrylate (HEMA) was spin-coated at $1500 \mathrm{rpm}$ for $30 \mathrm{~s}$, and RIE again performed with the previous conditions (Figure 1c). Ti and Au thin films were deposited with an electron-beam evaporator (ZZS550, MASTEK). $300 \AA$ of Ti was deposited at a rate of 2 $\AA / \mathrm{s}$ to increase the contact force between gold and PDMS. Then, $3000 \AA \mathrm{Au}$ was deposited at a rate of $3 \AA$ As.

To facilitate etching of the thin metal film, a mask was fabricated using a negative photoresist (DNR-300-40, DONGJIN, Korea) (Figure 1d). 
The photoresist was spin-coated onto the Au thin film at $1300 \mathrm{rpm}$ for $20 \mathrm{~s}$, and then exposed to $365-\mathrm{nm}$ illumination at an energy of $150 \mathrm{~mJ} / \mathrm{cm} 2$ and developed for $40 \mathrm{~s}$ to obtain a mask (Figure 1e).

The sample was immersed in an iodine-potassium iodide solution to etch the gold and titanium using buffered oxide etchant (BOE). After the etching process was complete, the photoresist was removed with acetone (Figure 1f).

High sacrificial posts were fabricated to expose the electrodes to the outside and control the electrode wall slope. The negative photoresist used in this study can form a film having a thickness of about $7 \mu \mathrm{m}$ during each spin-coating process. It was spincoated first at $1300 \mathrm{rpm}$ for $20 \mathrm{~s}$ and heat-treated at $95{ }^{\circ} \mathrm{C}$ for $90 \mathrm{~s}$. Again, a second spincoating was performed at the same rate, and then heat treatment was carried out at $110^{\circ} \mathrm{C}$ for $160 \mathrm{~s}$ to obtain a photoresist film having a thickness of about $20 \mu \mathrm{m}$.

Then, after exposure to an energy of about $150 \mathrm{~mJ} / \mathrm{cm} 2$, heat treatment was performed at $100{ }^{\circ} \mathrm{C}$ for $2 \mathrm{~min}$. Through this process, we could obtain the desired mask. Because of the increased thickness of the photoresist, if the exposure energy used is the same as that for a single film, the under-exposure condition can be induced. The slope of the sacrificial posts can be adjusted by under-exposure (Figure 1g).

To increase the bonding strength between PDMS and PDMS, an oxygen plasma treatment was performed. Then, a thin PDMS film with $25-\mu \mathrm{m}$-thickness was formed by spin-coating PDMS at $1500 \mathrm{rpm}$ for $90 \mathrm{~s}$ (Figure 1h).

The PDMS film was fabricated to cover the sacrificial posts, and the electrode and the pad region were then revealed by etching with a TBAF solution and pyrrolidone in a volume ratio of 1:10 (Figure 1i).

The outer shape of the sensor was then cut using a ND:YVO4 laser (3500-Smart PS, DPSS), with $354.7 \mathrm{~nm}$ wavelength, $2.7 \mathrm{~W}$ intensity, and $5 \mathrm{~mm} / \mathrm{s}$ velocity (Fig 1i). The laser pulse frequency was $20 \mathrm{kHz}$, with a pulse width of $5 \mu \mathrm{s}$.

Finally, the sample was stored in de-ionized (DI) water for 1 day to remove various chemicals.

\section{B. Simulation of Current Density Distribution}

The current density distribution according to the structure of the stimulating electrode was simulated mathematically [15], to determine the current density above the electrode using finite element analysis (COMSOL 4.3).

Three different electrode structures were modeled as shown in Figure 2. The electrode size, depth, and wall slope were set to $100 \mu \mathrm{m}, 10 \mu \mathrm{m}$, and $45^{\circ}$, respectively like Figure 2 . The physical parameters of the materials used in the simulations are as follows. Electrical conductivity $(\mathrm{S} / \mathrm{m})$ of PDMS, $\mathrm{Au}$, and $0.9 \% \mathrm{NaCl}$ are $2.75,4.103 \times 107$, and 1.6, respectively; the corresponding values of relative permittivity are $1.0 \times 10-13,7.8 \times 1011$, and 78 (Table 1).

Table 1. Simulation Parameters

\begin{tabular}{lll}
\hline Materials & Electrical Conductivity $(\mathrm{S} / \mathrm{m})$ & Relative Permittivity \\
\hline PDMS & 2.75 & $1.0 \times 10^{-13}$ \\
Gold & $4.103 \times 10^{7}$ & $7.8 \times 10^{11}$ \\
$0.9 \% \mathrm{NaCl}$ & 1.6 & 78 \\
\hline
\end{tabular}

The stimulation voltage was set at $35 \mathrm{mV}$. The in vivo environment was assumed to be $0.9 \%$ saline solution. 
a

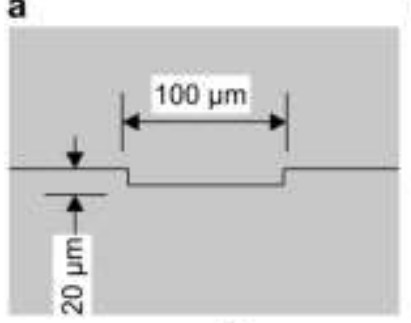

C b

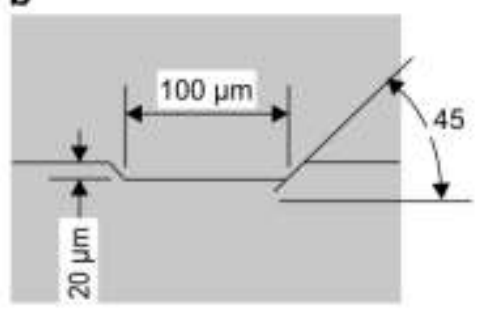

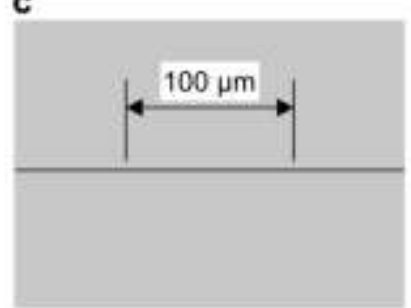

Figure 2. Three Types of Electrode Structure: (a) Vertical-Edge Electrode, (b) Slanted-Edge Electrode, and (c) Surface-Mounted Electrode

\section{Electrode Reliability Test}

Leakage is a frequent cause of failure in polymer-based electrodes [18]. Thus, accelerated life tests were performed in order to investigate failure of the stimulation electrodes due to leakage. The electrode was immersed in PBS solution at 40, 60, and $80^{\circ} \mathrm{C}$. The signal and ground of the signal generator were connected to adjacent electrodes, and the signal was a $5 \mathrm{~V}$ square wave in Figure 3. When an electrical leakage path occurs, crosstalk is generated between the electrodes, and the independence of the electrode is lost.

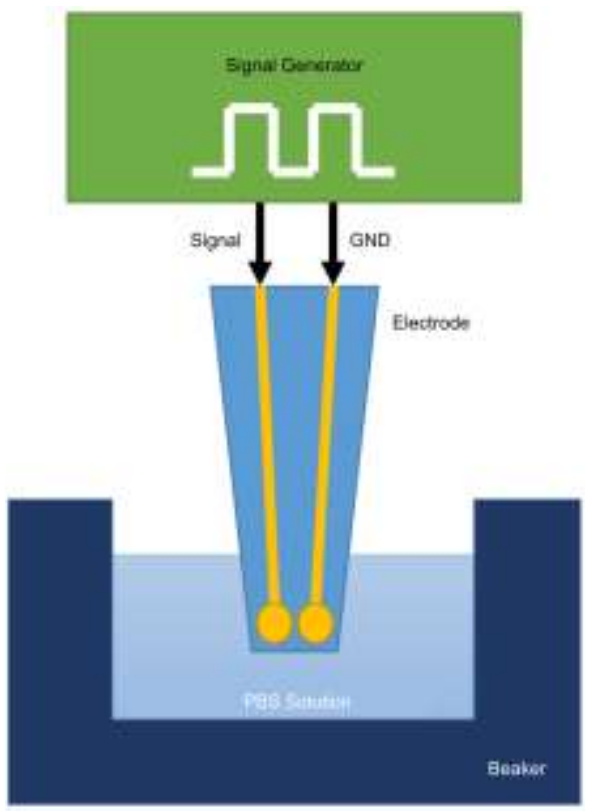

Figure 3. Reliability Test Environment of Proposed Electrode. The MultiElectrode was Immersed in PBS Solution, and a 5-V Square-Wave Signal was Applied 


\section{Result and Disscussion}

\section{A. Fabrication of Slanted-edge Electrode}

When fabricating sacrificial posts, there are two factors that affect the slope of the sidewall: the distance between the sample and the mask, and the amount of exposure energy. The amount of light diffraction varies according to the distance between the mask and the sample, which can be used to control the inclination of the photoresist structure. By adjusting the exposure time, the exposure energy of the upper and lower portions of the photoresist can be varied, because it takes time for the light to penetrate the photoresist. To adjust the mask distance, a $160-\mu \mathrm{m}$-thick PET film was used between the mask and photoresist layer. By adjusting the exposure time and the exposure distance, it is possible to manufacture sacrificial posts having various inclination angles[12].

Figure 4 shows the fabricated sacrificial posts. It can be seen that there is a difference in the size of the upper and lower patterns of the sacrificial posts, and the size of the lower part is noticeably smaller than that of the upper part.

a

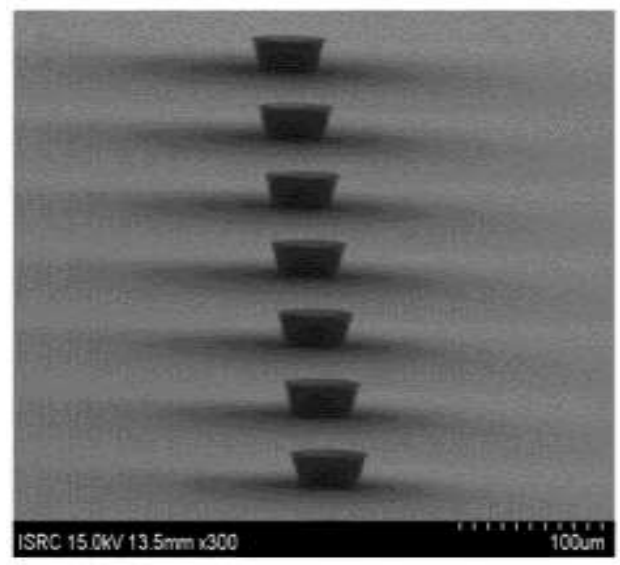

b

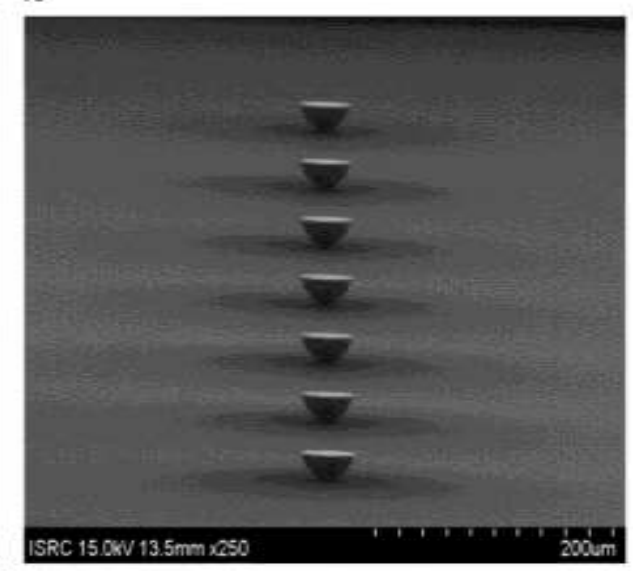

Figure 4. SEM Image[12] (a) Shows the Result of Photolithography using under-exposure only, and the angle of wall was about $80^{\circ}$; (b) shows the Result of Photolithography using Under-Exposure and an Exposure Interval of $160 \mu \mathrm{m}$, and the Angle of the Wall was about $50^{\circ}$

Figure 5 shows the PDMS hole according to the shape of the sacrificial posts.

a

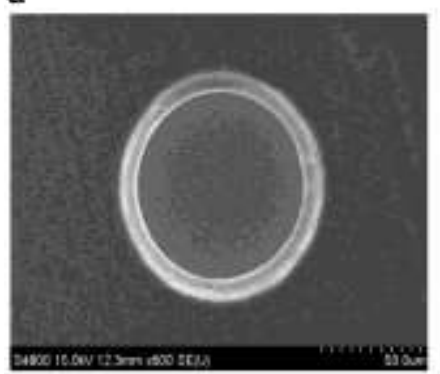
b

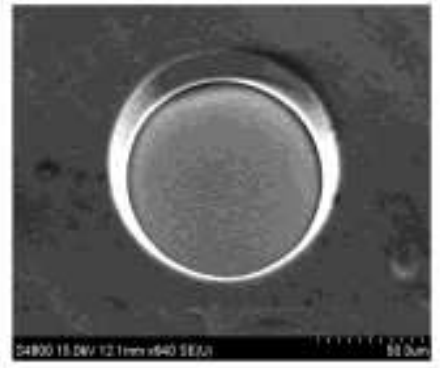

c

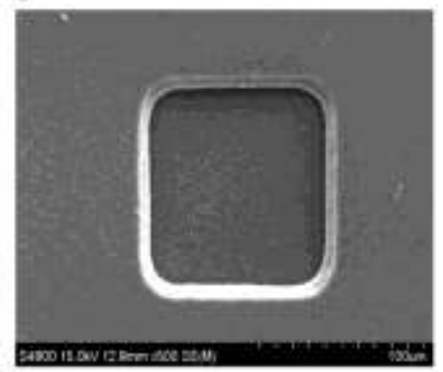

Figure 5. Slanted-edge Holes Created using the Sacrificial Post Method: After PDMS was Spin-Coated on the Sloped Sacrificial Posts, the Photoresist was removed to obtain a Slanted-Edge Hole. (a) Circular Electrode with a Diameter of $100 \mu \mathrm{m}$; (b) the Oblique View of (a); (c) Square Electrode with $100 \mu \mathrm{m}$ Side Length 
Figure 6(a) shows PDMS holes with vertical-edges fabricated using dry etching, Figure 6(b) shows slanted-edges fabricated using the photoresist casing scheme in Figure 1, and Figure 6(c) shows a surface-mounted electrode. All three types can be fabricated with PDMS using the proposed technique. The slanted-edge electrodes are advantageous for nerve tissue stimulation, and the surface-mounted electrodes can be in close contact with tissues, and are thus beneficial for nerve signal recording.

a

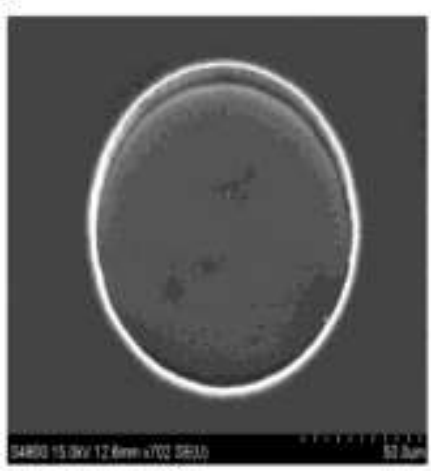

b

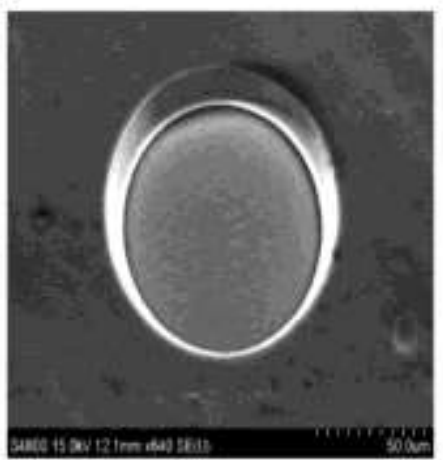

C

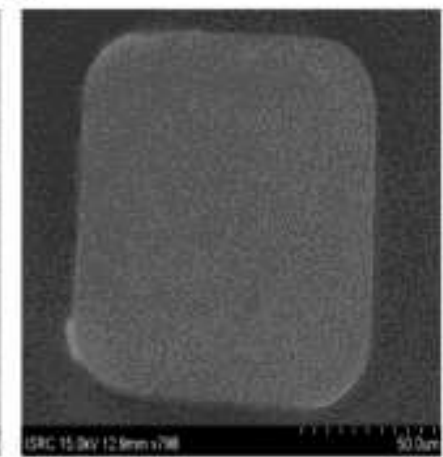

Figure 6. Three Types of Electrodes Fabricated using the Proposed Scheme: (a) Vertical-Edge Electrode; (b) Slanted-Edge Electrode; and (c) Surface-Mounted Electrode

Figure 7(a) shows an optical microscope image of the slanted-edge electrode; it can be seen that the gold electrode was located under the PDMS layer. However, the slope of the PDMS hole was not shown clearly on the optical microscope, and thus it was imaged again using SEM. Figure 7(b) clearly shows that the PDMS hole pattern has a slope. Figure 7(c) shows the electrode profile obtained using a profiler (Nanospec AFT/200, KLA-TENCOR, USA). The distance between points A-B was $\sim 17 \mu \mathrm{m}$, and that between points B-C was $\sim 90 \mu \mathrm{m}$. The PDMS film was 3-4 $\mu \mathrm{m}$ thicker in the vicinity of the electrode walls (point D), as compared to other locations, where it is considered that the PDMS film is affected by the underlying photoresist structure.

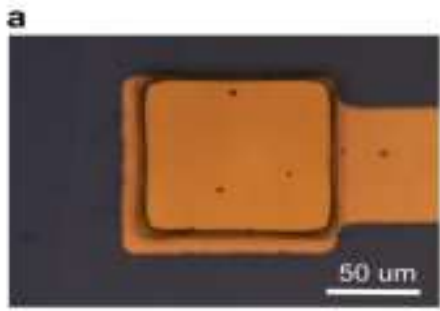

b
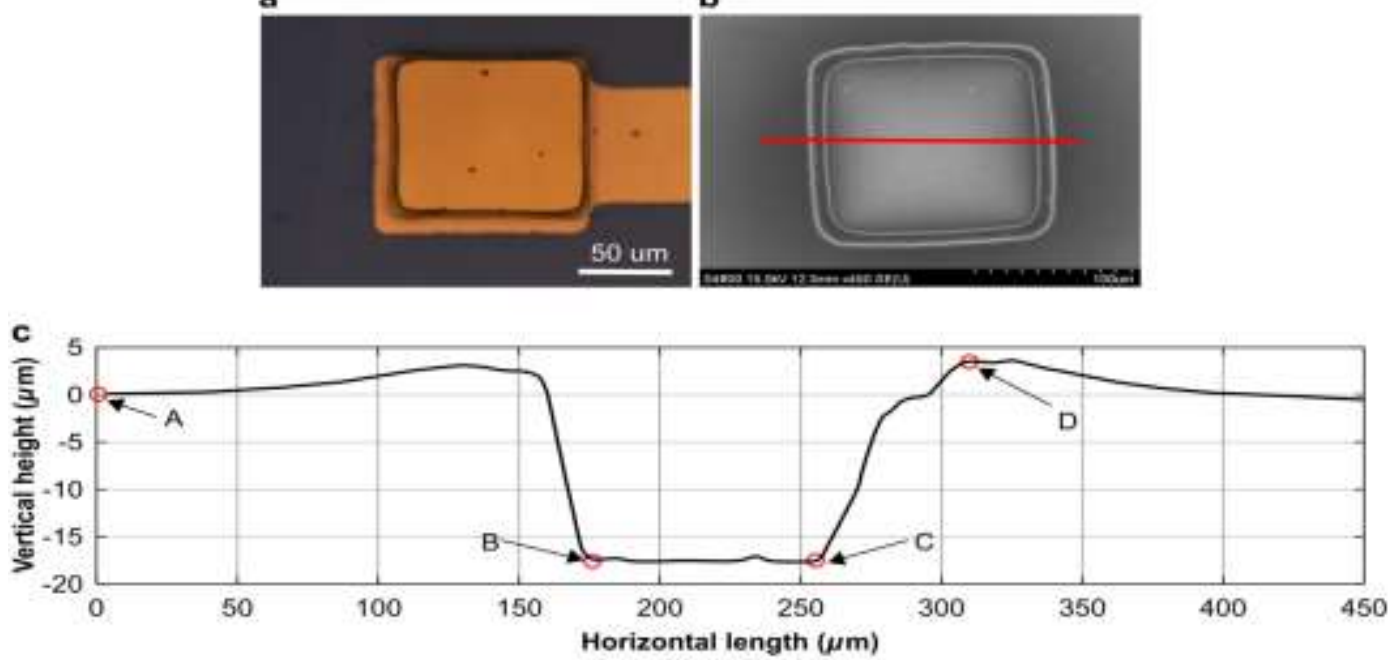

Figure 7. Electrode Fabrication Results using the Proposed Process: (a) Optical Microscope Image of Electrode; (b) SEM image; and (c) Electrode Profile Recorded along the Red Line in (b) 


\section{B. Simulation of Current Density Distribution}

Figure 8 shows COMSOL simulation results of current density distribution for the three electrode shapes. Figure 8(a) shows the simulation results of the conventional vertical-edge electrode, where high current density occurs at the sharp edges at the top of the electrode walls. In particular, this region is directly connected to the tissue when the electrode array is attached to the nerve tissue. In this case, the nerve tissue connected to the central region of the electrode is stimulated more weakly than the desired strength, but that at the edge region of the electrode is strongly stimulated, which may cause tissue damage. Figure 8(b) shows the simulation results of the slanted-edge electrode, where a high current density is formed at the bottom edge of the slanted walls. The current density distribution at the top surface that contacts the nerve tissue is relatively uniform compared to that for the vertical-edge electrodes. Figure 8(c) shows the simulation results for the surface-mounted electrode; the current density is very high at the edges of the electrode. The surface-mounted electrode interfaces directly with the nerve tissue; therefore, similar problems may occur as with the vertical-edge electrodes.
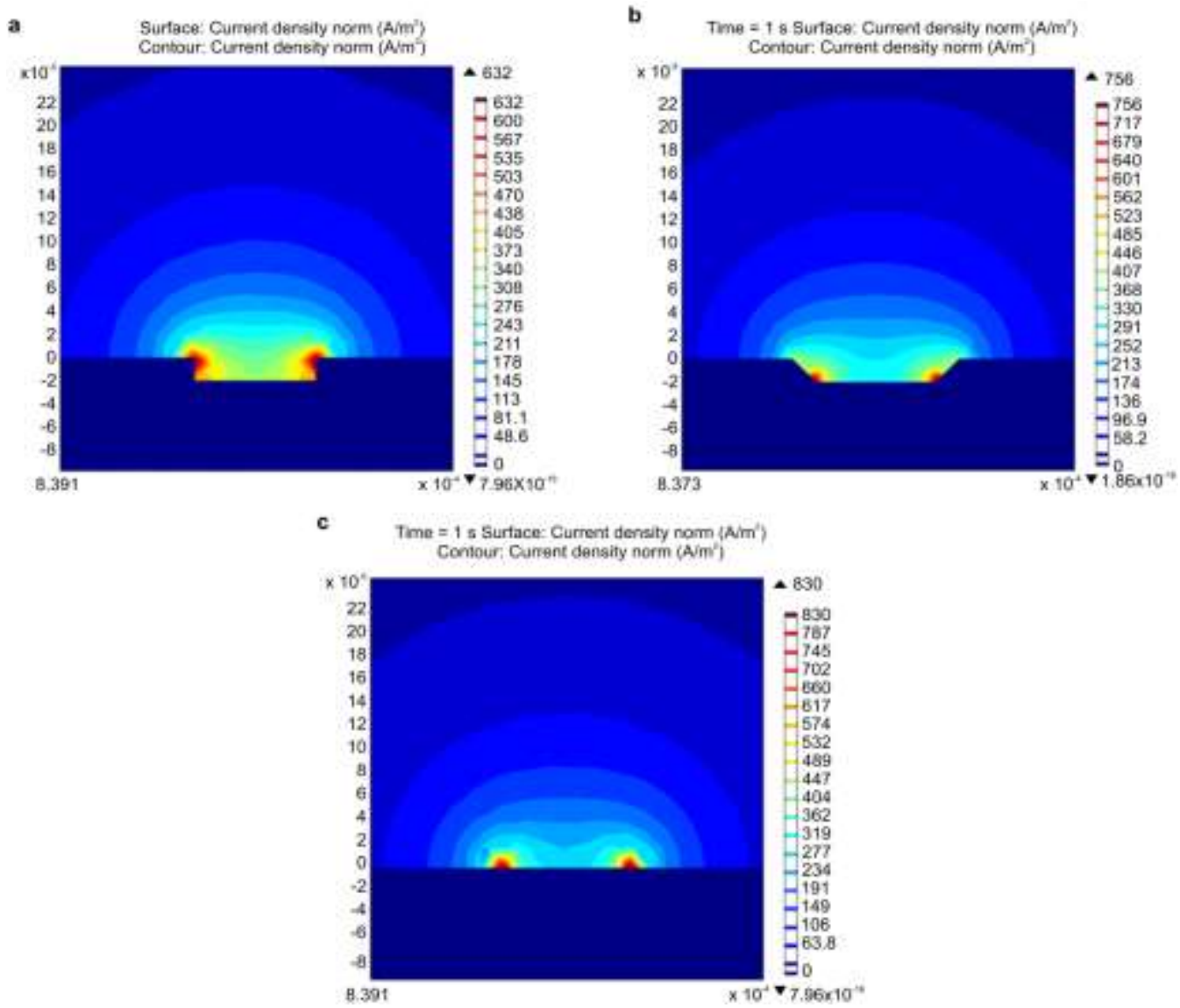

Figure 8. Current Density Distributions of 3 Types of Electrode: (a) VerticalEdge Electrode, (b) Slanted-Edge Electrode, and (c) Surface-Mounted Electrode. Red Color Represents High Current Density

The simulation results can be used to determine the level of stimulation at the electrode surface. It was found that the current density distribution had the same tendency as previously reported $[14,15]$. In addition, if the current density is high in a particular region of an electrode, corrosion may occur at that region [15]. Thus, by using slantededge electrodes, both tissues and electrodes could be protected. 

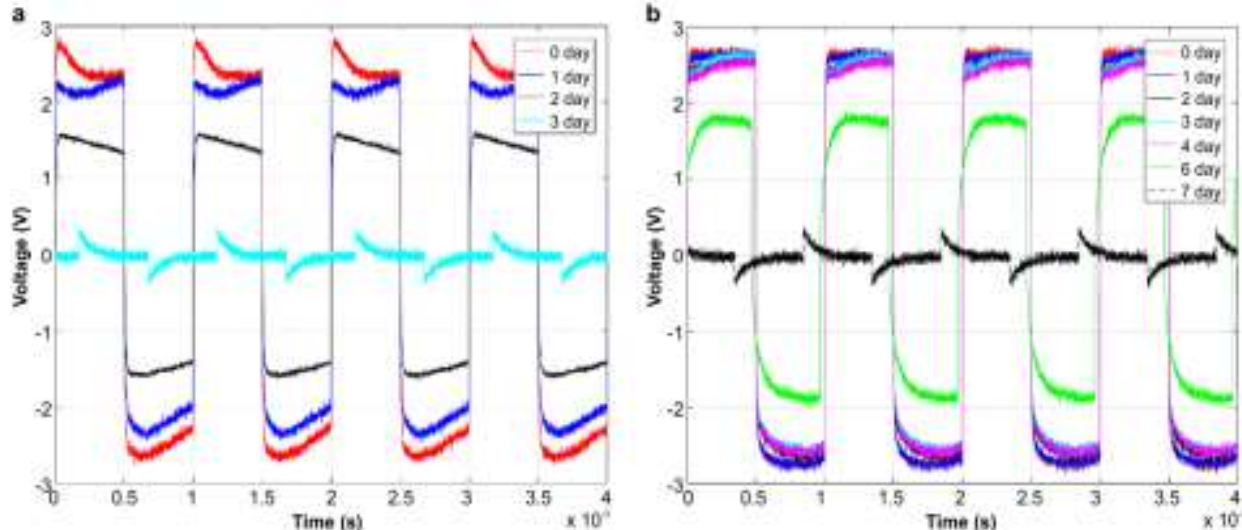

Figure 9. Leakage Current Test of the Vertical-Edge and Slanted-Edge Electrode in $80^{\circ} \mathrm{C}$ PBS Solution: (a) Vertical-Edge Electrode Worked for 2 Days; (b) Slanted-Edge Electrode Worked for 6 Days

The results of the in vitro reliability test are shown in Fig 9. The vertical-edge electrode showed leakage from the 2nd day, whereas the slanted-edge electrode showed leakage from the 6th day. The accelerated lifetime test causes any degradation to occur at a faster rate than under the actual use conditions. The lifetime can be mathematically expressed as [19]:

$$
t_{0}=A_{F} t_{S},
$$

where $t \_0$ is the lifetime in the use condition, $t \_s$ is the lifetime in the accelerated condition, and AF is the acceleration factor. The Arrhenius model is the most widely used relational expression for accelerated life tests relying on temperature [19]:

$$
\tau=A e^{E / k T},
$$

where $\tau$ is the failure time, $\mathrm{E}$ is the activation energy $(\mathrm{eV}), \mathrm{k}$ is the Boltzmann constant in $\mathrm{eV}, \mathrm{T}$ is the absolute temperature, and $\mathrm{A}$ is a constant. Thus, Equation 2 can be used to calculate the acceleration factor (AF). The $\mathrm{AF}$ was found to be 3.43 at $60^{\circ} \mathrm{C}$ and 10.25 at $80^{\circ} \mathrm{C}$ for the slanted-edge electrode.

Based on this result, assuming that the temperature of the human body is close to $40^{\circ} \mathrm{C}$, it can be predicted that the slanted-edge electrode will operate for about 2 months and the vertical-edge electrode will operate for about 20 days. Therefore, the developed PDMS electrodes cannot be used as an implanted electrode, but can be used for acute experiments.

\section{Conclusions}

Multi-channel electrodes can be used to stimulate targeted neurons with electrical current. The conventional vertical-edge electrode exhibits maximum current density at the edge of the electrode, and a non-uniformity of the current density distribution with a low current density at the center. This phenomenon can lead to irreversible cell damage during neural stimulation and electrode damage in certain areas of the electrode. Therefore, an electrode structure with more uniform current density distribution is required, which can be achieved by slanted-edge electrodes[12].

A PDMS hole with a gentle slope was fabricated on the electrode by applying the proposed PDMS patterning technique using sacrificial posts. In consideration of 
biocompatibility, the proposed electrodes use gold as an electrode material, which has a low ionization tendency and high biocompatibility, so as not to cause toxicity due to corrosion[12].

Studies on the electrode structure have been performed in terms of current density distribution, and leakage test showed that lifetime can be elongated with a more uniform current density distribution, which is achieved for slanted-edge electrodes. However, it is necessary to verify the electrode performance through animal experiments in the future.

\section{Acknowledgments}

This work was supported by the National Research Foundation of Korea (NRF) grant funded by the Korean government (Ministry of Education) (No. NRF2017R1D1A1B03028351).

\section{References}

[1] L. F. Nicolas-Alonso and J. Gomez-Gil, "Brain computer interfaces, a review, Sensors", doi:10.3390/s120201211, vol. 12, no. 2, (2012), pp. 1211-1279.

[2] W. He, G. C. McConnell, T. M. Schneider and R. V. Bellamkonda, "A novel anti-inflammatory surface for neural electrodes", Adv. Mater, doi:10.1002/adma.200700943, vol. 19, no. 21, (2007), pp. 35293533.

[3] K. C. Cheung, "Implantable microscale neural interfaces", Biomed. Microdevices, doi:10.1007/s10544006-9045-z, vol. 9, no. 6, (2007), pp. 923-938.

[4] R. F. Labadie, R. Balachandran, J. H. Noble, G. S. Blachon, J. E. Mitchell, F. A. Reda, B. M. Dawant and J. M. Fitzpatrick, "Minimally invasive image-guided cochlear implantation surgery: First report of clinical implementation", Laryngoscope, doi:10.1002/lary.24520, vol. 124, no. 8, (2014), pp. 1915-1922.

[5] M. Taketani and M. Baudry, "Advances in Network Electrophysiology", Springer, New York, (2006).

[6] M. HajjHassan, V. Chodavarapu and S. Musallam, "NeuroMEMS: neural probe microtechnologies", Sensors, doi:10.3390/s8106704, vol. 8, no. 10, (2008), pp. 6704-6726.

[7] C. Hassler, T. Boretius and T. Stieglitz, "Polymers for neural implants", J. Polym. Sci. Part B: Polym. Phys., doi:10.1002/polb.22169, vol. 49, no. 1, (2011), pp. 18-33.

[8] K. C. Cheung, P. Renaud, H. Tanila and K. Djupsund, "Flexible polyimide microelectrode array for in vivo recordings and current source density analysis", Biosens. Bioelectron., doi:10.1016/j.bios.2006.08.035, vol. 22, no. 8, (2007), pp. 1783-1790.

[9] N. Chou, S. Yoo and S. Kim, "A largely deformable surface type neural electrode array based on PDMS”, IEEE Trans. Neural Syst. Rehabil. Eng., doi:10.1109/TNSRE.2012.2210560, vol. 21, no. 4, (2013), pp. 544-553.

[10] L. Guo, K. W. Meacham, S. Hochman and S. P. DeWeerth, "A PDMS-based conical-well microelectrode array for surface stimulation and recording of neural tissues", IEEE Trans. Biomed. Eng., doi:10.1109/TBME.2010.2052617, vol. 57, no. 10, (2010), pp. 2485-2494.

[11] S. E. Lee, S. B. Jun, H. J. Lee, J. Kim, S. W. Lee, C. Im, H.-C. Shin, J. W. Chang and S. J. Kim, “A flexible depth probe using liquid crystal polymer", IEEE Trans. Biomed. Eng., doi:10.1109/TBME.2012.2196274, vol. 59, no. 7, (2012), pp. 2085-2094.

[12] S. J. Hwang, D. J. Oh, P. G. Jung, S. M. Lee, J. S. Go, J.-H. Kim, K.-Y. Hwang and J. S. Ko, "Dry etching of polydimethylsiloxane using microwave plasma", J. Micromech. Microeng., doi:10.1088/0960-1317/19/9/095010, vol. 19, no. 9, (2009), pp. 95010.

[13] J. M. Kim and S. H. Kim, "Fabrication of PDMS-based Slanted Edge-type Microelectrode Array", Advanced Science and Technology Letters, vol. 146, (FGCN 2017), pp. 144-147.

[14] J. Guimont and B. Ziaie, "A batch-manufacturable uniform current density metallic-shell hemispherical microelectrode”, Conf. Proc. IEEE Eng. Med. Biol. Soc., doi:10.1109/IEMBS.2004.1404157, vol. 2, (2004), pp. 4149-4152.

[15] D. A. Ksienski, "A minimum profile uniform current density electrode", IEEE Trans. Biomed. Eng., doi:10.1109/10.142643, vol. 39, no. 7, (1992), pp. 682-692.

[16] J. T. Rubinstein, F. A. Spelman, M. Soma and M. F. Suesserman, "Current density profiles of surface mounted and recessed electrodes for neural prostheses", IEEE Trans. Biomed. Eng., doi:10.1109/TBME.1987.326007, vol. 11, (1987), pp. 864-875.

[17] J. D. Wiley and J. G. Webster, "Analysis and control of the current distribution under circular dispersive electrodes”, IEEE Trans. Biomed. Eng., doi:10.1109/TBME.1982.324910, vol. 5, (1982), pp. 381-385.

[18] W. F. Agnew, D. B. McCreery, T. G. H. Yuen and L. A. Bullara, "Local anaesthetic block protects against electrically-induced damage in peripheral nerve", J. Biomed. Eng., vol. 12, no. 4, (1990), pp. 301-308. 
[19] J. Viventi, D.-H. Kim, L. Vigeland, E. S. Frechette, J. A. Blanco, Y.-S. Kim, A. E. Avrin, V. R. Tiruvadi, S. W. Hwang, A. C. Vanleer and D. F. Wulsin, "Flexible, foldable, actively multiplexed, high-density electrode array for mapping brain activity in vivo", Nat. Neurosci., doi:10.1038/nn.2973, vol. 14, no. 12, (2011), pp. 1599-1605.

[20] W. B. Nelson, "Accelerated Testing: Statistical Models", Test Plans, and Data Analysis, Wiley, Hoboken, NJ, (2004).

\section{Authors}

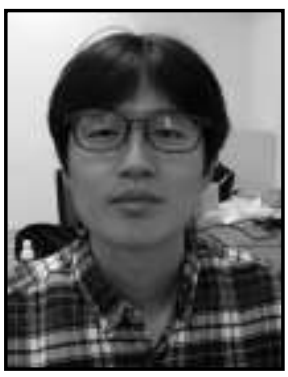

Jun-Min Kim, received the B.S. and M.S. degrees in the Department of Electrical Engineering and Computer Science, College of Engineering, Seoul National University (SNU), Seoul, Korea, in 2008 and 2010, respectively, where he is currently working toward the $\mathrm{Ph} . \mathrm{D}$. degree.

His current research interests include the development of new generation polymer-based biosignal recording electrode based on closed-loop system (neural stimulation and recording electrodes and circuitries), and low cost neural prosthetic systems.

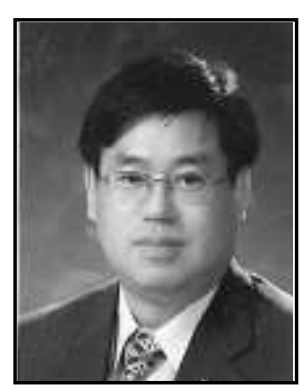

Sun-Hyung Kim, received his B.S., M.S. and Ph.D. degrees in Electronic Engineering from Sungkyunkwan University, Korea, in 1979, 1981 and 1988, respectively. Since 1989, He has been a professor in Department of Information and Communication Engineering, Soonchunhyang University.

From 2005 to the Present, he was a vice-chairman of Korea University Invention Association. From 2013 to the Present, he was a vice-chairman of Korea Institute of Information Technology.

His research interests include Data Communication, Embedded system, Network, etc. 
International Journal of Control and Automation

Vol. 10, No. 12 (2017) 